\title{
MHD equilibrium analysis with anisotropic pressure in LHD
}

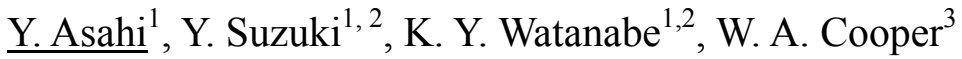 \\ 1 The graduate University for Advanced Studies, Toki, Japan \\ 2 National Institute for Fusion Science, Toki, Japan \\ 3 PPCL Lausanne, Lausanne Switzerland
}

\begin{abstract}
The MHD equilibrium theory predicts that anisotropic plasma pressures parallel and perpendicular to field lines are not flux surface quantities. The method to identify the distortion of pressure from the surface average in experimental plasmas is not established. Here, the effects of the distortion on the measured values are investigated in the Large Helical Device (LHD). The MHD equilibria with the anisotropic pressure are studied using a 3D MHD equilibrium code, ANIMEC, which uses the bi-Maxwellian model as an anisotropic plasma pressure model in the 3D geometry. We study the position of magnetic axis and the magnetic flux obtained by magnetic diagnostic as candidates of characteristic parameter in the MHD equilibrium. As the results, we find that the position of magnetic axis is not sensitive to the distortion from the flux surface average, but the magnetic flux is sensitive to the distortion. This suggests that the magnetic diagnostics has a possibility to estimate the distortion from flux surface average.
\end{abstract}

\section{Introduction}

High beta plasmas with more than 5\% volume averaged beta in LHD experiments are generated and maintained only by tangentially injected neutral beams (NB). According to the evaluation of the beam pressure, which is based on a Monte-carlo analysis, $30 \%$ of the total plasma pressure corresponds to the contribution from the fast beam pressure ions [1]. The reason such a relatively high beam fraction is due to the conditions of high beta discharges with the low density and the low magnetic field. The high energy ions from the tangential NB are well confined even when the magnetic field is low. Because of the long slowing down time of high energy particles in low density regimes and the small thermal pressure due to the low magnetic field, the beam pressure cannot be ignored compared with the thermal pressure. As a result, it is expected to cause an anisotropy in the pressure with parallel component along the equilibrium magnetic field lines $p_{\|}$greater than $p_{\perp}$, the perpendicular component.

The MHD equilibrium theory with the anisotropic pressure predicts that the pressure is not the flux surface quantity. The evaluation of the magnitude of pressure distortion from the 
flux surface average due to the anisotropy is an important subject to identify the MHD equilibrium. Recently, a three dimensional MHD equilibrium analysis code was developed [2], in which a bi-Maxwellian model was implemented in the VMEC code to take anisotropic pressure into account. In this paper, the effects of the distortion from the flux surface average on the magnetic axis positions and the magnetic flux observed in the magnetics are investigated. We refer to a saddle type flux loop which placed at the bottom of the torus [3], and the values of magnetic flux are estimated from the equilibrium current obtained by ANIMEC. For $p_{\perp}>p_{\|}$plasmas, the distortion from the flux surface quantity are explained in Sec. 2 , the change of the axis position and the flux in the effect of trapped particles are shown in Sec. 3, and a summary is in Sec. 4.

\section{Pressure profile distortion from the flux surface average}

The bi-Maxwellian distribution function

$$
F_{h}(s, \varepsilon, \mu)=N(s)\left(\frac{m_{h}}{2 \pi T_{\perp}(s)}\right)^{\frac{2}{3}} \exp \left[-m_{h}\left(\frac{\mu B_{c}}{T_{\perp}(s)}+\frac{\left|\varepsilon-\mu B_{c}\right|}{T_{\|}(s)}\right)\right]
$$

was implemented to energetic ions in the ANIMEC code. Where $s$ is the radial index, $N(s)$ is the density amplitude factor, $\varepsilon$ is the kinetic energy, $m_{\mathrm{h}}$ is the mass of the high-energy particles, and $T_{\|}$ and $T_{\perp}$ are the temperatures of high energy particles in the direction parallel and perpendicular to magnetic field. The value $T_{\perp} / T_{\|}$is related to the pressure anisotropy. $B_{\mathrm{c}}$ is a threshold field strength which prescribe the magnitude
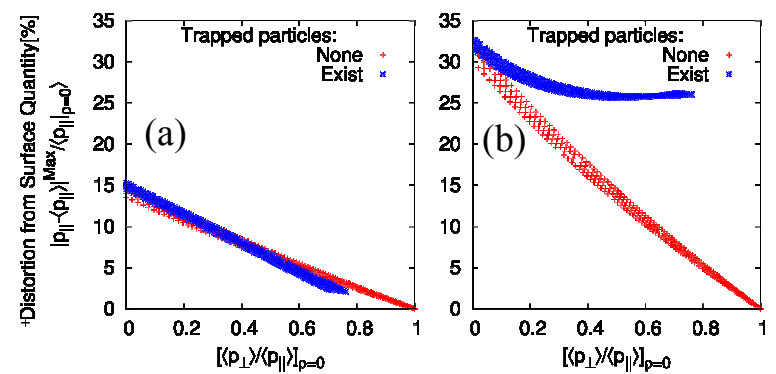

Fig.1 The maximum absolute value of the difference between the parallel pressure and its flux surface average normalized to its average value on axis (a) and the corresponding maximum absolute value for the perpendicular pressure (b) as a function of the ratio of $\left\langle p_{\perp}\right\rangle$ to $\left\langle p_{\|}\right\rangle$corresponding to the strength of the anisotropy at the magnetic axis[6].

of trapped particles fraction. The trapped particles are assumed to exist in the surfaces which include smaller field than $B_{\mathrm{c}}$, and the change of the distribution function, comparing to the purely passing particle case, is significant [5]. Even when $T_{\perp} / T_{\|}=1$, the pressure with trapped particles remain anisotropy, whereas $T_{\perp} / T_{\|}=1$ corresponds to isotropy in the case without trapped particles. In our simulations, two cases are considered. One is the case without trapped particles, and another is the case with trapped particles. Fig.1 shows the magnitude of pressure profile distortion 
from its surface average [6]. For the case with trapped particle, the ratio of the surface averaged pressure $p_{\perp}$ to $p_{\|}$at the axis $\left.\left\langle p_{\perp}>\mid<p_{\|}\right\rangle\right|_{\rho=0}$ does not be unity even when $T_{\perp} / T_{\|}=1$, and the difference of pressure from its averaged value remain considerable, whereas the distortion decrease as $\left\langle p_{\perp}>/<p_{\|}>\left.\right|_{\rho}=0\right.$ decrease in the case without trapped particle.

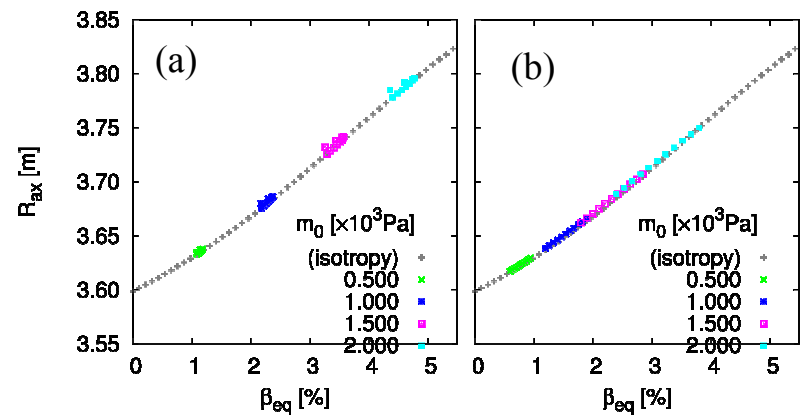

Fig.2 The dependence of the magnetic axis position obtained by numerical analysis with a model which trapped particles does not exist(a) and exist(b). The gray points correspond to the isotropic pressure plasma axis positions which $T_{\perp} / T_{\|}=1[6]$.

\section{Effects of the trapped particles on the axis position and the magnetic flux}

First of all, we define the equilibrium beta value which expected to yields the equivalent magnetic axis shift to the isotropic pressure equilibria with the same beta value. Helical plasma equilibria with anisotropic pressure were previously studied [7]. With CGL formula $\mathbf{p}=p_{\perp} \mathbf{I}+\left(p_{\|}-p_{\perp}\right) \mathbf{n n}$ and a low $\beta$ ordering, the Pfirsch-Schlüter current can be expressed as

$$
j_{P S}=\frac{\rho}{b^{*}}\left(P_{\|}^{\prime}+P_{\perp}^{\prime}\right) \cos \theta
$$

where $\rho=r / a,{ }^{\prime}=\partial / \partial \rho, \mathrm{P}_{\|}$and $\mathrm{P}_{\perp}$ are the leading terms of $p_{\|}$and $p_{\perp}$ and are functions of only the magnetic flux surface and $\theta$ is the poloidal angle. Other variables used in the Eq.(2) are defined in Ref.[7]. Focusing on the dependence of the Pfirsch-Schlüter current on the pressure components, the equivalent beta is defined as

$$
\beta_{e q}=\frac{\int d V\left(p_{\|}+p_{\perp}\right)}{2 \int d V\left(B^{2} / 2 \mu_{0}\right)}
$$

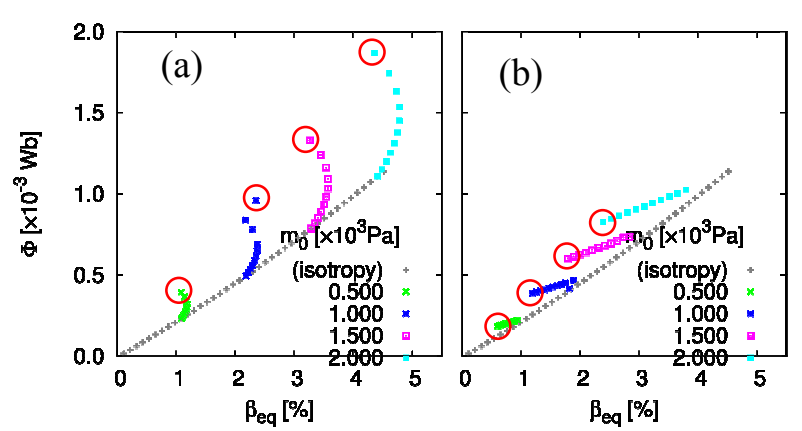

Fig. 3 The dependence of the magnetic flux obtained by numerical analysis with a model which trapped particles does not exist(a) and exist(b). The gray points correspond to the isotropic pressure plasma axis positions which $T_{\perp} / T_{\|}=1$.

In this simulations, the pressure profile $M(s)$ is given as $M(s)=m_{0}(1-s)$ where $s=\rho^{2}$. $m_{0}$ is the pressure at magnetic axis. The points with green, blue, pink, light blue colored correspond to the different value $m_{0}=0.5,1.0,1.5,2.0\left[\times 10^{3} \mathrm{~Pa}\right]$ with $T_{\perp} / T_{\|}=[0,1]$. Fig.2 shows the axis position analyzed in two models. One is the case without trapped particles and another is the case with trapped particles in the region smaller field than $B_{\mathrm{c}}=0.60[\mathrm{~T}]$ in 
Eq.(1). It should be noted the field strength at the magnetic axis is 0.425 [T]. All of the points in Figs.2 ( $a, b)$ nearly coincides to the isotropic case.

In the next, we focused on the effects against magnetic flux. Fig. 3 shows the estimated saddle loop flux calculated from equilibrium current obtained by ANIMEC analysis[4]. The saddle loop is placed at the bottom of the LHD torus, and can detect the vertical field generated by Pfirsch-Schlüter current. The points housed in red circle represents the results with $T_{\perp} / T_{\|}=0$. The magnetic flux varies with the trapped particles exist or not, namely the difference in the shape of velocity distribution and the value deviates from the isotropic case. The saddle loop flux seems to detect the effect of the distortion from the surface average, and therefore, the flux could be a candidate of estimating the distortion.

\section{Summary}

By modeling anisotropic plasmas with the bi-Maxwellian, the distortion of parallel and perpendicular pressure from its surface average are estimated with $p_{\|}>p_{\perp}$ case. The distortion, particularly for $p_{\perp}$ was strongly affected by whether the trapped particles exist or not. To establish the method of identifying the pressure anisotropy and the shape of velocity distribution, the response of the magnetic axis position and the magnetic flux by the pressure anisotropy were examined in LHD magnetic field configurations. For the magnetic axis, we obtain the results that the position is depending only on $\beta_{\mathrm{eq}}$, regardless of the amount of trapped particles or the pressure anisotropy. This means the distinction of the two factors only by an axis position is impossible. In contrast, the magnetic flux vary whether the trapped particles exist or not, or in other words, the difference in the shape of velocity distribution prescribed by Bc in Eq.(1) even in the same $\beta_{\text {eq. }}$. This result suggests that the saddle loop measurement can detect the distortion of pressure from its surface average. The influence of pressure anisotropy and the shape of velocity distribution on a diamagnetic measurement or the Pfirsch-Schlüter current profile is one of the future subjects.

This study is supported by the project oriented course budget NIFS10ULPP022.

\section{References}

[1] K. Y. Watanabe, et al. Fusion Science and Technology 58, 160 (2010).

[2] W. A. Cooper, et al. Comp. Phys. Comm. 180, 1524 (2009).

[3] S. Sakakibara, et al. Fusion Science and Technology 58, 176 (2010).

[4] T. Yamaguchi, et al. Plasma Physics and Controlled Fusion 48, L73 (2006).

[5] W. A. Cooper, et al. Nuclear Fusion 46, 683(2006).

[6] Y. Asahi, et al. Plasma and Fusion Research (to be published).

[7] W. N. G. Hitchon, et al. Nuclear Fusion 23, 383 (1983). 